\title{
Injury pattern simulation and mapping of complex tibial plateau fractures that involve the posterior plateau with three-dimensional computed tomography
}

\author{
Shuo Pan, A-Qin Peng, Ya-Ning Hu, Shuai Wang, Yan-Long Zhang, Yong Wang \\ Department of Orthopedic Surgery, The Third Hospital of Hebei Medical University, Shijiazhuang, China \\ Contributions: (I) Conception and design: AQ Peng; (II) Administrative support: AQ Peng, S Pan; (III) Provision of study materials or patients: AQ \\ Peng, S Pan, YN Hu, S Wang; (IV) Collection and assembly of data: AQ Peng, S Pan, YN Hu, S Wang; (V) Data analysis and interpretation: S Pan, \\ YN Hu, S Wang, YL Zhang, Y Wang; (VI) Manuscript writing: All authors; (VII) Final approval of manuscript: All authors. \\ Correspondence to: A-Qin Peng, MD, PhD. Department of Orthopedic Surgery, The Third Hospital of Hebei Medical University, No. 139 Ziqiang \\ Road, Shijiazhuang 050051, China. Email: pengaqin666@163.com.
}

Background: Tibial plateau fractures involving the posterior plateau (TPFIPs) are complex intra-articular fractures that are difficult to stabilize. Understanding the characteristics of these fractures together with the injury pattern is beneficial for surgeons to choose an optimal treatment strategy. However, the complicated morphology and injury patterns of TPFIPs are poorly characterized. The purpose of this retrospective study was to investigate the injury patterns and fracture characteristics of complex TPFs by applying threedimensional (3D) simulation and fracture mapping methods.

Methods: In total, 171 TPFIPs were retrospectively reviewed, and the injury pattern was simulated and analyzed by applying a 3D method with Mimics software, which allowed matching of the fractured articular surfaces of the tibial plateau to the femoral condyle surface. The major articular fracture lines were mapped and then superimposed on a template. The tibial motion angle after fracture injury pattern simulation and the major fracture line angle were quantitatively analyzed, while the injury patterns and fracture characteristics were qualitatively analyzed.

Results: Four main injury patterns with distinctive fracture characteristics were observed in this study. In total, 72 TPFs exhibited extension as the pattern of injury with a split posterolateral fragment, and 61 fractures exhibited the flexion-internal rotation injury pattern; compression was the main feature of posterolateral fractures. Furthermore, 21 fractures exhibited the flexion-external rotation injury pattern, with a small posteromedial fragment, and 17 fractures exhibited the flexion-neutral injury pattern, with both parts of the posterior plateau fracture and anterior dislocation being observable. The major articular fracture line angles were significantly different between the four main injury patterns $\left(85.92^{\circ}, 46.79^{\circ}, 148.26^{\circ}\right.$, and $16.21^{\circ}$, median values, $\mathrm{P}<0.05)$. Two injury patterns, namely, flexion-internal rotation and flexion-external rotation, exhibited rotation in the axial plane $\left(24.13^{\circ} \pm 8.33^{\circ},-15.13^{\circ} \pm 5.14^{\circ}, \mathrm{P}<0.05\right)$.

Conclusions: In this study, a method involving a simulated injury pattern was developed and combined with evaluations of fracture characteristics, including two-dimensional (2D) and 3D analyses, to comprehensively describe both the morphologies and injury patterns of TPFIPs.

Keywords: Tibial plateau fractures (TPFs); injury patterns; fracture characteristics; 3D CT

Submitted Jun 30, 2020. Accepted for publication Nov 16, 2020.

doi: $10.21037 / \mathrm{atm}-20-5043$

View this article at: http://dx.doi.org/10.21037/atm-20-5043 


\section{Introduction}

Complex tibial plateau fractures (TPFs) involving the posterior region of the tibial plateau are immensely challenging to treat, even for experienced orthopedists. The fracture morphological characteristics are complex, the fracture location is difficult to detect on $\mathrm{X}$-ray, and posterior TPFs are difficult to stabilize with conventional surgical approaches and instruments. Failure to identify and manage a complex TPF can lead to stiffness, pain, instability and traumatic arthritis of the knee (1-4). The prevention of such disability is of substantial importance and requires a thorough understanding of the fracture characteristics and injury pattern to choose the optimal surgical strategy.

Many systems have been developed to help orthopedists understand fracture morphological characteristics. Based on computed tomography (CT), posterior plateau TPFs were described by Luo (5) as posterior column fractures; this definition was then revised by Chang (6) and divided into posterolateral and posteromedial fractures. However, the roles of such systems in understanding the actual fracture characteristics remain limited and a matter of debate. Molenaars (7) characterized TPFs with a fracture mapping technique to describe the actual fracture characteristics in a two-dimensional (2D) template; Kfuri and Schatzker (8) further revised the classic classification with a threedimensional (3D) method, which included posterior plateau fractures, instead of X-ray views, which contributed to the new understanding of surgeons regarding fracture characteristics. However, the understanding of injury patterns is limited. Several studies have described the morphological characteristics of posterolateral and/or posteromedial TPFs and assume that the injury pattern involves the axial loading of force on the knee in the flexion position (9-11). However, describing the morphology of TPFs only partially is insufficient for surgical planning, and the current injury pattern is also insufficient for interpreting the complexity of TPFs involving the posterior plateau (TPFIPs) with various fracture morphologies.

It is essential for surgeons to know the pattern of an injury to correctly understand a fracture, make a treatment decision, and choose an optimal surgical strategy. TPFs are caused by impact on the femoral condyle. The morphologies of the fractured articular surface and the femoral condyle may match at certain positions; thus, the position of the knee at the time of fracture is believed to be the injury pattern. To date, however, the injury patterns of actual complex fractures have most often been reconstructed utilizing imagination and speculation, which requires extensive experience. In this study, a novel 3D CT simulation technology was applied to simulate and analyze the injury patterns of complex TPFIPs, and the characteristics of these fractures were depicted using a fracture mapping method. It was hypothesized that the simulation and mapping of complex TPFs would reveal multiple recurrent injury patterns with distinctive fracture characteristics. We present the following article in accordance with the STROBE reporting checklist (available at http://dx.doi.org/10.21037/atm-20-5043).

\section{Methods}

\section{Subjects}

A retrospective search of an orthopedic database maintained at a level I trauma center was performed to obtain the CT imaging data of patients treated for TPFs from December 2012 to December 2018. The study was conducted in accordance with the Declaration of Helsinki (as revised in 2013) and was approved by our hospital institutional review board (IRB protocol \#2019-036-1). Informed consent was waived because of the retrospective nature of the study. All patients in the database who had a TPFIPs and a complete set of Digital Imaging and Communications in Medicine (DICOM) files generated by CT were included in this retrospective analysis. Patients younger than 18 years old or with pathological fractures, previous knee surgery and/ or existing knee ligament malfunctions, or CT images of insufficient quality (e.g., axial CT images with a slice thickness less than $3 \mathrm{~mm}$ and CT scans not showing intact femoral condyles) were excluded. The 171 TPFIPs included were assigned a Schatzker classification with $\mathrm{X}$-ray and CT.

DICOM files were imported into Mimics software (19.0, Materialise, Leuven, Belgium) to create a project file for each patient. The overall threshold was set at $225 \mathrm{HU}$ in all cases. First, the orientation, including the axial, sagittal, and coronal planes, was standardized with the "Reslice" function to ensure that the sagittal plane was perpendicular to the posterior femoral condylar axis, the coronal plane was perpendicular to the anterior-posterior femoral condylar axis, and the axial plane was perpendicular to the sagittal and coronal planes. Then, the tibial and femoral masks were created separately using the "CT Bone Segmentation" function to automatically separate the target bone from surrounding structures. The tibial mask contained the tibia and fibula as a whole. After adjusting the view orientation, 3D objects of the tibia and femur were reconstructed separately from the masks using "Calculate 3D by Mask", and the optimal quality was chosen 

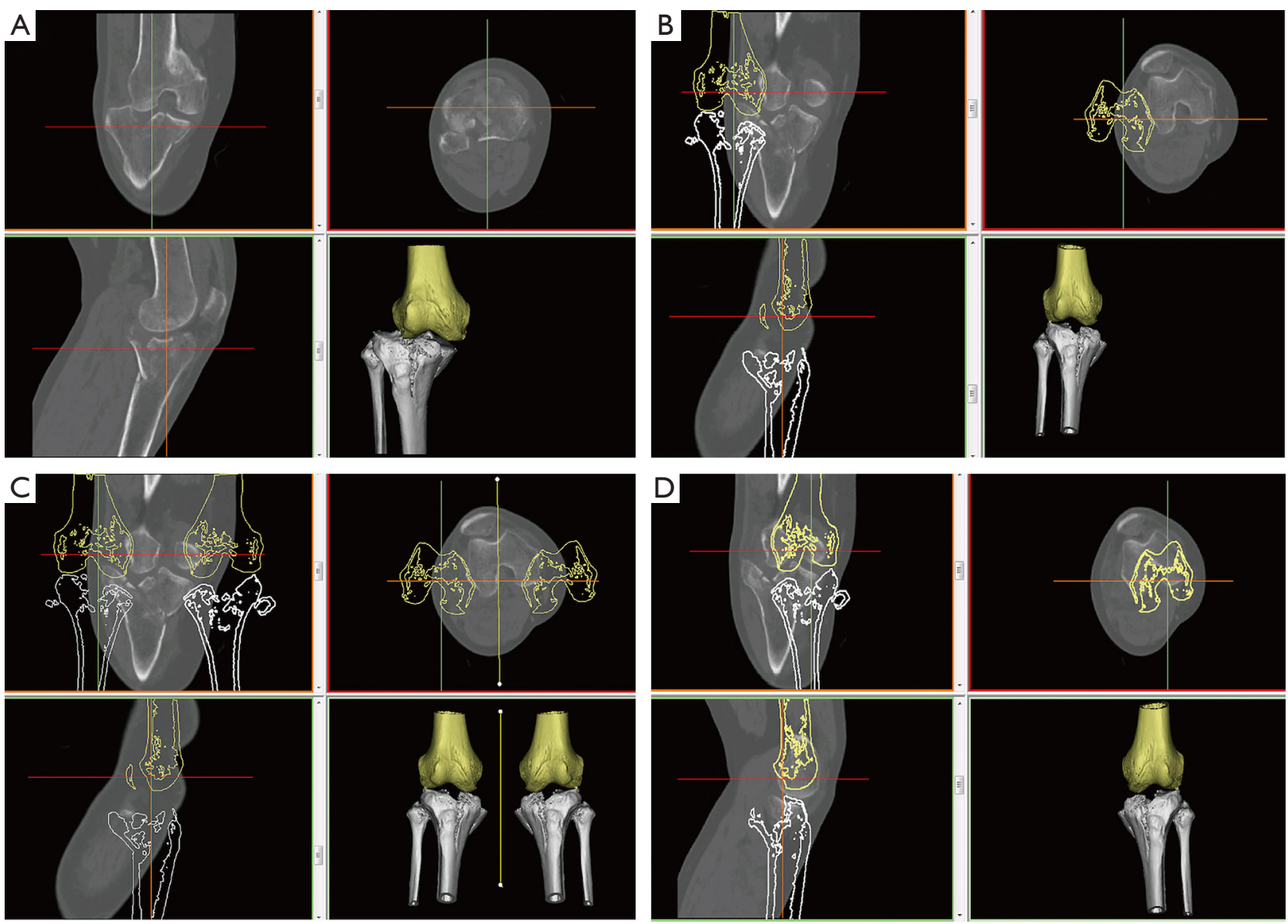

Figure 1 The creation of the 3D objects for tibial and femur. (A) CT DICOM files were imported into Mimics software to create a project file for each patient. Masks of the tibia and femur were created separately, and 3D models of each mask were then reconstructed. (B) The 3D models of the tibia and femur were adjusted to full extension as the reference position using the "Pan" and "Rotate" functions if necessary. The orientations of the axial, sagittal, and coronal planes were standardized to ensure that the sagittal plane was perpendicular to the posterior condylar axis, the coronal plane was perpendicular to the anteroposterior condylar axis, and the axial plane was perpendicular to both the sagittal and coronal planes. (C) For injury position analysis and fracture mapping of the tibia, the 3D model of the right limb was flipped horizontally to obtain a left limb model. (D) A left extensional limb model was created.

with a smooth filter of "1". The positions of the 3D tibia and femur objects were adjusted using the "Pan" and "Rotate" functions to be fully extended relative to the reference position $\left(0^{\circ}\right.$ in the three planes). For injury pattern analysis and fracture mapping, the 3D models of the right limb were flipped horizontally in the coronal planes using the "mirror" function to obtain left limb models (Figure 1A,B,C,D).

\section{Injury pattern analysis}

To simulate the injury pattern during fracture, we moved (translated and rotated) the tibial 3D object to ensure that the articular surface of the TPF matched that of the femoral condyles using the "Reposition" function. During repositioning, we also scrolled through the images in the three 2D-view windows to ensure that the articular contour lines of the tibia and femur matched in all slices and planes. When the geometric forms between the articular surfaces were matched in all three planes and $3 \mathrm{D}$ views, the optimal match was achieved, and the final injury pattern was recorded. All matching processes were performed by orthopedists experienced in TPF management and knowledgeable of knee biomechanics and kinematics. The final injury pattern (tibial position) for each fracture was 

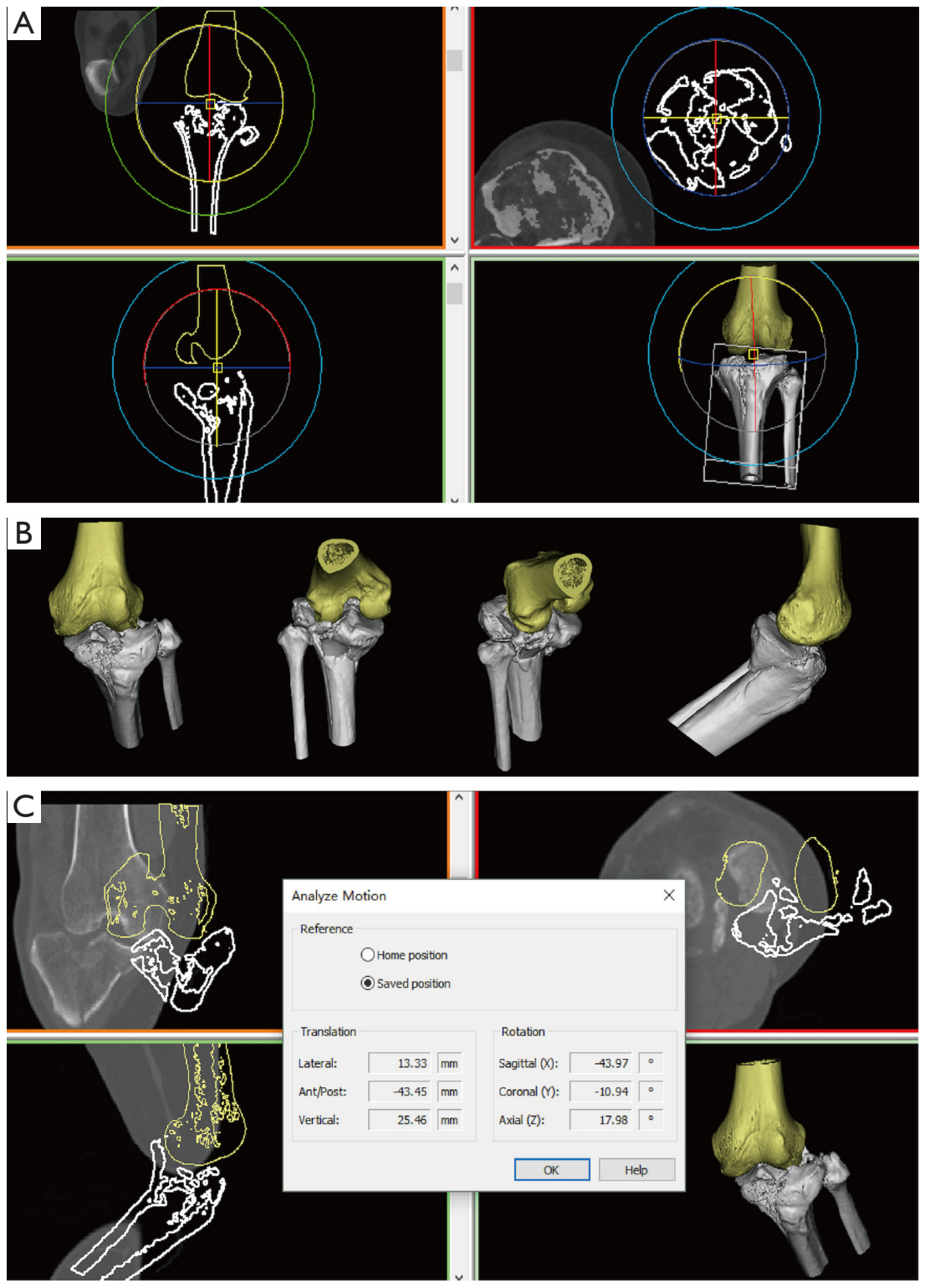

Figure 2 Injury pattern simulation and analysis. (A) The tibial rotational center point was moved to the center of the knee joint. (B) The tibial 3D object was moved (translated and rotated) to ensure that the articular surface of the tibial plateau fracture matched that of the femoral condyle using the "Reposition" function. (C) The 2D images were examined in three dimensions to ensure that the match was optimal on all slices. The translation and rotation values were calculated by the software. "Translation" items represent the direction of tibial displacement, and the values represent the degree of displacement. The data of the 3 items in "Rotation" represent the tibial position change relative to the extension (reference) position in the three planes. A positive value in the sagittal plane represents extension, while a negative value represents flexion; in the axial plane, a positive value represents internal rotation, while a negative value represents external rotation; in the coronal plane, a positive value represents valgus, while a negative value represents varus.

determined by consensus between two senior orthopedic surgeons. The data of tibial motion in the three planes calculated by the "Analyze Motion" function of the software were recorded and analyzed (Figure $2 A, B, C$ ). In the sagittal plane, a final tibial position degree greater than $-15^{\circ}$ was defined as an extension pattern, and a position less than $-15^{\circ}$ was defined as a flexion pattern. In the axial plane, a degree greater than $10^{\circ}$ was defined as an internal rotation pattern, 

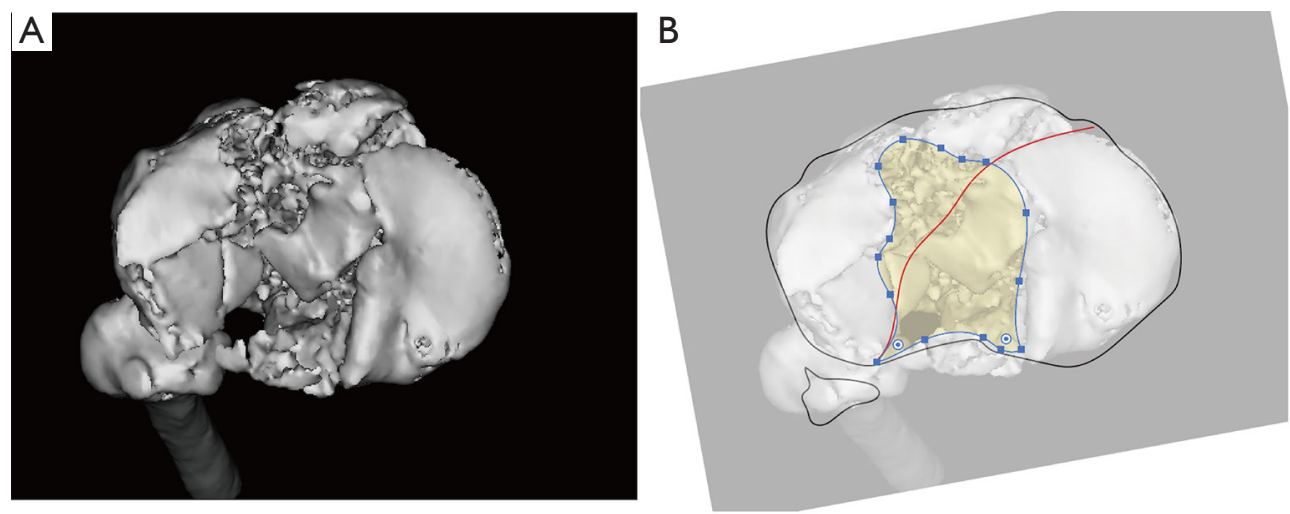

C

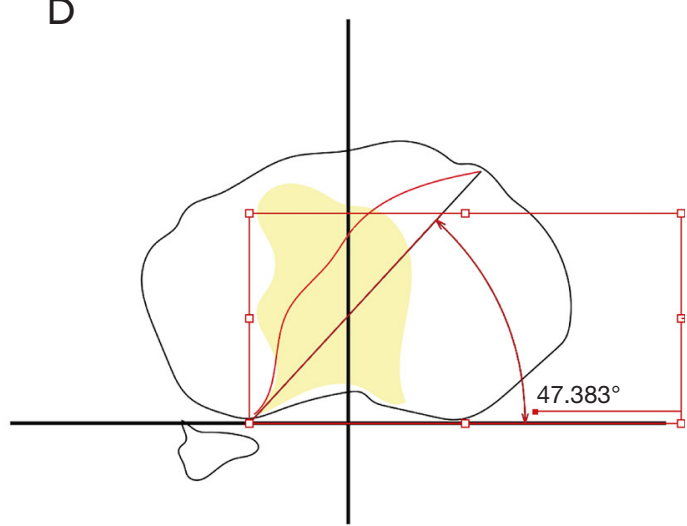

Figure 3 Fracture mapping and major articular fracture line analysis. The top view images of the 3D tibial models (A) were imported on a template. (B,C) Fracture mapping. Specific tibial plateau landmarks, such as the medial and lateral tibial plateaus, the tibial tubercle, and the fibula, were matched to ensure proper rotation and alignment. The MFL (red line) and comminution area (yellow area) were drawn on the template based on the 3D models. (D) The MFLA $\left(47.383^{\circ}\right.$ ) was determined as the angle between the MFL and the reference line (the line between the lateral and medial posterior condyles of the plateau) clockwise by measuring.

a degree less than $-10^{\circ}$ was defined as an external rotation pattern, and a degree between $10^{\circ}$ and $-10^{\circ}$ was defined as a neutral pattern. In the coronal plane, the boundary point was defined as $0^{\circ}$ for a valgus $\left(>0^{\circ}\right)$ or varus $\left(<0^{\circ}\right)$ pattern.

\section{Fracture mapping}

The fracture mapping method was first described by Armitage (12) and modified by Molenaars (7) for TPFs. In this study, intra-articular images of TPFs were obtained using 3D models instead of CT slices for fracture mapping. In the 3D reference position (extension position), the tibial object was selected, and the "top" button for selection of the top view of the tibial plateau articular surface, which is perpendicular to the medullary cavity of the tibia. The top-view image of the $3 \mathrm{D}$ model was imported into Adobe Illustrator software (2019, Adobe Systems Incorporated, San
Jose, CA, USA) on a 2D standard template of an intact left tibial plateau. Major articular fracture lines (MFLs) were identified based on the $3 \mathrm{D}$ models and drawn on the $2 \mathrm{D}$ template, and the MFL angle (MFLA) was determined to be the angle between the MFL and the horizontal reference line clockwise by measuring using the CAD function (Figure 3).

The lateral and medial contact areas in the tibial plateau where the femoral condyles impinged were identified from the $3 \mathrm{D}$ injury pattern view. The contact area on the plateau, fracture characteristics, morphologies of the posterior fragments and fracture lines were recorded and were descriptive in nature.

\section{Data analysis}

Patient characteristics and fracture measurement data are expressed as proportions or means and standard deviations. 
Table 1 Patient demographics and fracture characteristics

\begin{tabular}{|c|c|}
\hline Parameter & Patients $(n=168)$ \\
\hline Mean age [range] (yr) & $43.6[19-74]$ \\
\hline \multicolumn{2}{|l|}{ Sex, n (\%) } \\
\hline Male & $112(66.7)$ \\
\hline Female & $56(33.3)$ \\
\hline \multicolumn{2}{|l|}{ Side of injury, $\mathrm{n}(\%)$} \\
\hline Right & $70(41.7)$ \\
\hline Left & $95(56.5)$ \\
\hline Bilateral & $3(1.8)$ \\
\hline \multicolumn{2}{|c|}{ Schatzker classification $(\mathrm{N}=171), \mathrm{n}(\%)$} \\
\hline Type I & $4(2.3)$ \\
\hline Type II & $30(17.5)$ \\
\hline Type III & $27(15.8)$ \\
\hline Type IV & $37(21.7)$ \\
\hline Type V & $7(4.1)$ \\
\hline Type VI & $66(38.6)$ \\
\hline \multicolumn{2}{|l|}{ Concomitant injury, n (\%) } \\
\hline Fracture of proximal fibula & $80(46.8)$ \\
\hline Fracture of tibial shaft & $9(5.3)$ \\
\hline $\operatorname{MFLA}\left({ }^{\circ}\right)^{*}$ & $72.40 \pm 38.41$ \\
\hline
\end{tabular}

*, the values are given as the mean and standard deviation. MFLA, major fracture line angle.

The injury pattern (tibial motion), fracture mapping and MFLA were analyzed both quantitatively and qualitatively. The injury pattern "Rotation" data for the three planes and the MFLA are expressed as medians and interquartile ranges (IQRs). These values were compared using the KruskalWallis and Nemenyi tests. Significance was defined as $\mathrm{P}<0.05$, and statistical analyses were performed with SPSS software (version 25.0, IBM). The morphological fracture mapping analysis and characterization were descriptive in nature.

\section{Results}

\section{Subjects}

In total, four main injury patterns (extension, flexioninternal rotation, flexion-external rotation, and flexionneutral) and six subpatterns were identified in this series of TPFIPs. The main injury patterns were identified based on the tibial position in the sagittal and axial planes. In the extension injury pattern, according to the "screw-home" mechanism (13), no obvious axial rotation pattern was observed. In the flexion pattern, three types of axial rotation injury patterns were observed: flexion-internal rotation, flexion-external rotation, and flexion-neutral. Based on the tibial position in the coronal plane, the main injury patterns (except for the flexion-neutral pattern) were divided into the valgus $\left(>0^{\circ}\right)$ and varus $\left(<0^{\circ}\right)$ subpatterns.

Among the 171 complex TPFIPs, 98 (57.3\%) and 73 $(42.7 \%)$ fractures occurred in the left and right knees, respectively, with male patients predominating (112 male patients, including 3 with bilateral TPFs, compared with 56 female patients). Based on CT and X-ray examinations, 4 TPFs were classified as Schatzker type I (2.34\%), 30 as type II (17.54\%), 27 as type III (15.79\%), 37 as type IV (21.64\%), 7 as type V (4.09\%), and 66 as type VI (38.60\%). The demographics and baseline characteristics of the patients with TPFs are shown in Table 1 . The injury pattern criteria and fracture characteristics are shown in Table 2, the tibial position and MFLA data are shown in Table 3, and a diverse diagram of MFLs is shown in Figure 4.

\section{Extension injury pattern}

In total, $72(42.1 \%)$ TPFs exhibited an extension pattern $\left(-1.56^{\circ}\right.$ in the sagittal plane) with no obvious rotation $\left(0.77^{\circ}\right.$ in the axial plane) between the tibia and femur. Among them, 53 TPFs exhibited the valgus subpattern (extension-valgus subpattern), and 19 exhibited the varus subpattern (extension-varus subpattern) $\left(13.74^{\circ}\right.$ and $-13.85^{\circ}$, respectively, in the coronal plane, $\mathrm{P}=0.000)$. The $\mathrm{MFL}$ oriented from anterior to posterior (MFLA, $85.92^{\circ}$ ), which resulted from the femoral condyles impinging on the contact areas of the tibial plateau, located in the anterolateral quarter plateau and the central part of the medial plateau (Figure 5).

Lateral fractures were mainly compressed in the anterior part of the lateral tibial plateau (anterior to the fibular head), and the morphology of the posterolateral plateau was split. Medial plateau fractures split, and in severe cases, a transverse subfracture line originating from the middle region and extending in the medial and distal direction was observed (Figure 5).

\section{Flexion-internal rotation injury pattern}

In total, $61 \mathrm{TPF}$ exhibited a flexion and internal rotation pattern $\left(-41.52^{\circ}\right.$ in the sagittal plane and $24.24^{\circ}$ in the 
Table 2 Injury pattern criteria and fracture characteristics

\begin{tabular}{|c|c|c|c|c|c|c|c|}
\hline \multirow{2}{*}{ Parameter } & \multicolumn{2}{|c|}{ Extension } & \multicolumn{2}{|c|}{ Flexion-internal rotation } & \multicolumn{2}{|c|}{$\begin{array}{c}\text { Flexion-external } \\
\text { rotation }\end{array}$} & \multirow{2}{*}{$\begin{array}{c}\text { Flexion-neutral } \\
\text { N/A }\end{array}$} \\
\hline & Valgus & Varus & Valgus & Varus & Valgus & Varus & \\
\hline \multicolumn{8}{|l|}{ Rotation } \\
\hline Sagittal & \multicolumn{2}{|c|}{$>-15^{\circ}$} & \multicolumn{2}{|c|}{$<-15^{\circ}$} & \multicolumn{2}{|c|}{$<-15^{\circ}$} & $<-15^{\circ}$ \\
\hline \multicolumn{8}{|l|}{ Fracture characteristic } \\
\hline \multicolumn{8}{|l|}{ Lateral plateau } \\
\hline \multicolumn{8}{|l|}{ Anterolateral plateau } \\
\hline \multicolumn{8}{|l|}{ Posterolateral plateau } \\
\hline Fracture morphology & Split & Split & $\begin{array}{l}\text { Compress and } \\
\text { comminute }\end{array}$ & $\begin{array}{l}\text { Compress and } \\
\text { comminute }\end{array}$ & Split & Split & $\begin{array}{l}\text { Compress and } \\
\text { comminute }\end{array}$ \\
\hline Cortex fracture & Yes & $\begin{array}{c}\text { Yes } \\
\text { (type V and VI) }\end{array}$ & Yes & Yes & Yes & Yes & Yes \\
\hline \multicolumn{8}{|l|}{ Medial plateau } \\
\hline \multicolumn{8}{|l|}{ Anteromedial plateau } \\
\hline Cortex fracture & $\begin{array}{c}\text { Yes } \\
\text { (type V and VI) }\end{array}$ & Yes & Yes & Yes & No & No & No \\
\hline
\end{tabular}

axial plane). Among them, 50 TPFs exhibited the valgus subpattern (flexion-internal rotation valgus subpattern), and 11 exhibited the varus subpattern (flexion-internal rotation varus subpattern $)\left(10.86^{\circ}\right.$ and $-20.84^{\circ}$, respectively, in the coronal plane, $\mathrm{P}=0.000)$. With tibial flexion and internal rotation, the lateral contact area was located in the posterolateral quarter plateau, medial to the fibular head, and the medial contact area was located in the anterior area of the medial plateau; as a result, the MFL was oriented from the posterolateral to anteromedial plateau (MFLA, $46.79^{\circ}$ ).

The morphology of a posterolateral fracture was comminuted and compressed, and the fracture line extended distally from the back of the tibia, but the cortex of the anterolateral tibial plateau was intact. In the medial plateau, this fracture exhibited an inverted pyramid shape with anteromedial and distal tips, and the fracture was always accompanied by a small posterolateral part; an oblique fracture line was observable, and distal displacement was notable (Figure 6).

\section{Flexion-external rotation injury pattern}

In total, $21 \mathrm{TPF}$ exhibited a flexion and external rotation pattern $\left(-35.15^{\circ}\right.$ in the sagittal plane and $-13.81^{\circ}$ in the axial plane). Among them, 18 exhibited the valgus subpattern (flexion-external rotation valgus pattern), and 3 exhibited the varus subpattern (flexion-external rotation varus pattern) $\left(16.69^{\circ}\right.$ and $-10.52^{\circ}$, respectively, in the coronal plane, 
Table 3 Four main injury patterns characteristics

\begin{tabular}{|c|c|c|c|c|c|}
\hline Parameter & \multicolumn{4}{|c|}{ Injury pattern } & $P$ value \\
\hline $\mathrm{N}=171$ & 72 & 61 & 21 & 17 & \\
\hline $\operatorname{MFLA}\left({ }^{\circ}\right)^{*}$ & $85.92(23.48)$ & $46.79(7.38)$ & $148.26(9.49)$ & $16.21(10.88)$ & 0.000 \\
\hline \multicolumn{6}{|l|}{ Rotation $\left({ }^{\circ}\right)$} \\
\hline Coronal $^{*}$ & $11.32(25.65)$ & $9.54(5.16)$ & $15.84(5.75)$ & $-0.41(5.25)$ & 0.000 \\
\hline Axial $^{*}$ & $0.77(3.72)$ & $24.24(9.68)$ & $-13.81(3.58)$ & $1.74(4.77)$ & 0.000 \\
\hline \multicolumn{6}{|c|}{ Schatzker classification ( $N=171), n(\%)$} \\
\hline Type I & $2(2.78)$ & 0 & $1(4.76)$ & $1(5.88)$ & \\
\hline Type IV & $6(8.33)$ & $21(34.43)$ & $1(4.76)$ & $9(52.95)$ & \\
\hline Type V & $5(6.94)$ & 0 & $1(4.76)$ & $1(5.88)$ & \\
\hline Type VI & $46(63.89)$ & $14(22.95)$ & $6(28.57)$ & 0 & \\
\hline
\end{tabular}

*, the values are given as the median and inter quartile range (IQR). Kruskal Wallis and Nemenyi tests are applied for all group's comparison.

A
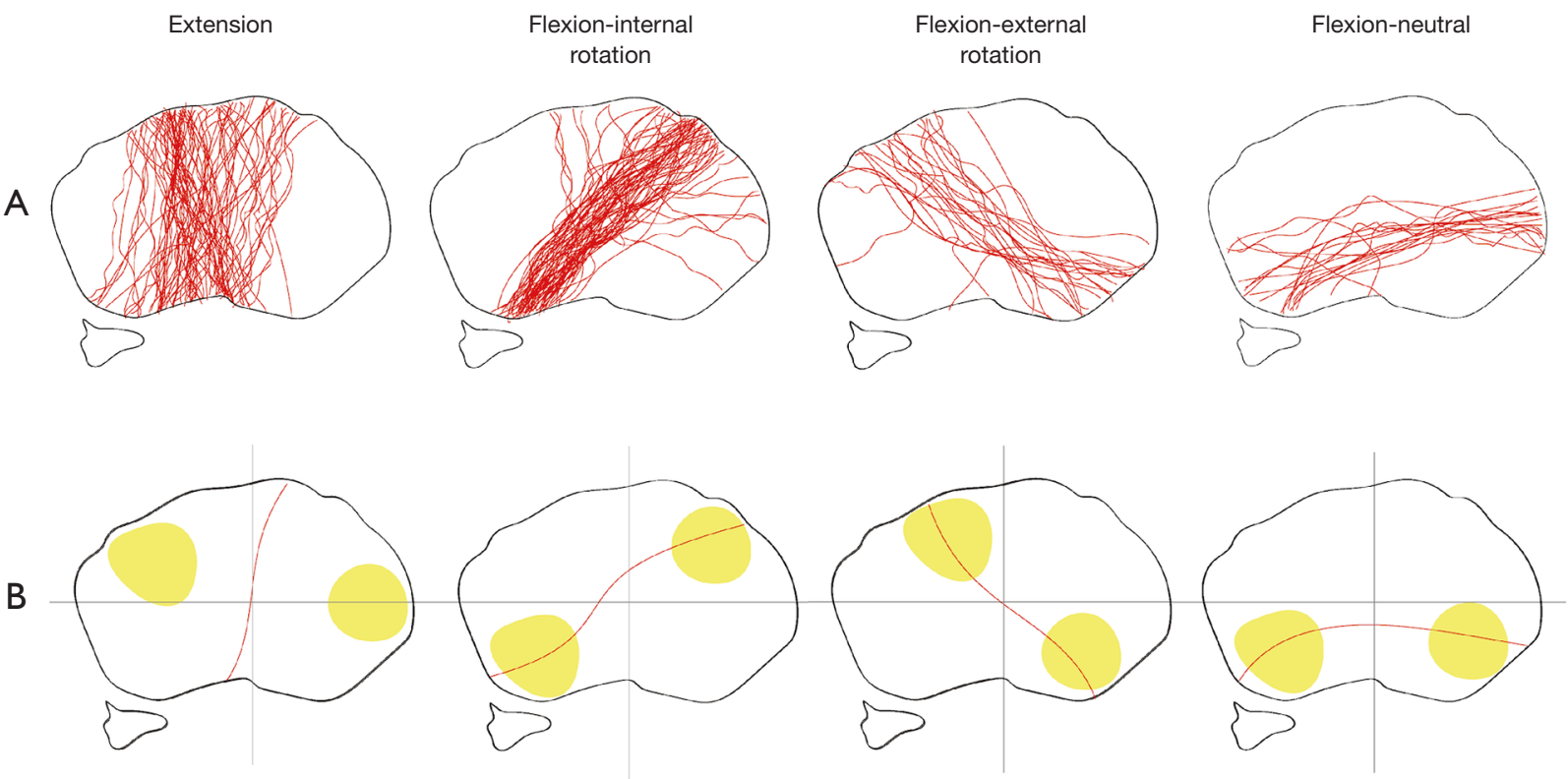

Figure 4 The MFLs mapping of the four main injury patterns. (A) The overlap of the MFLs in the four main injury patterns. (B) The contact areas between the tibial plateau and the formed fracture line. 


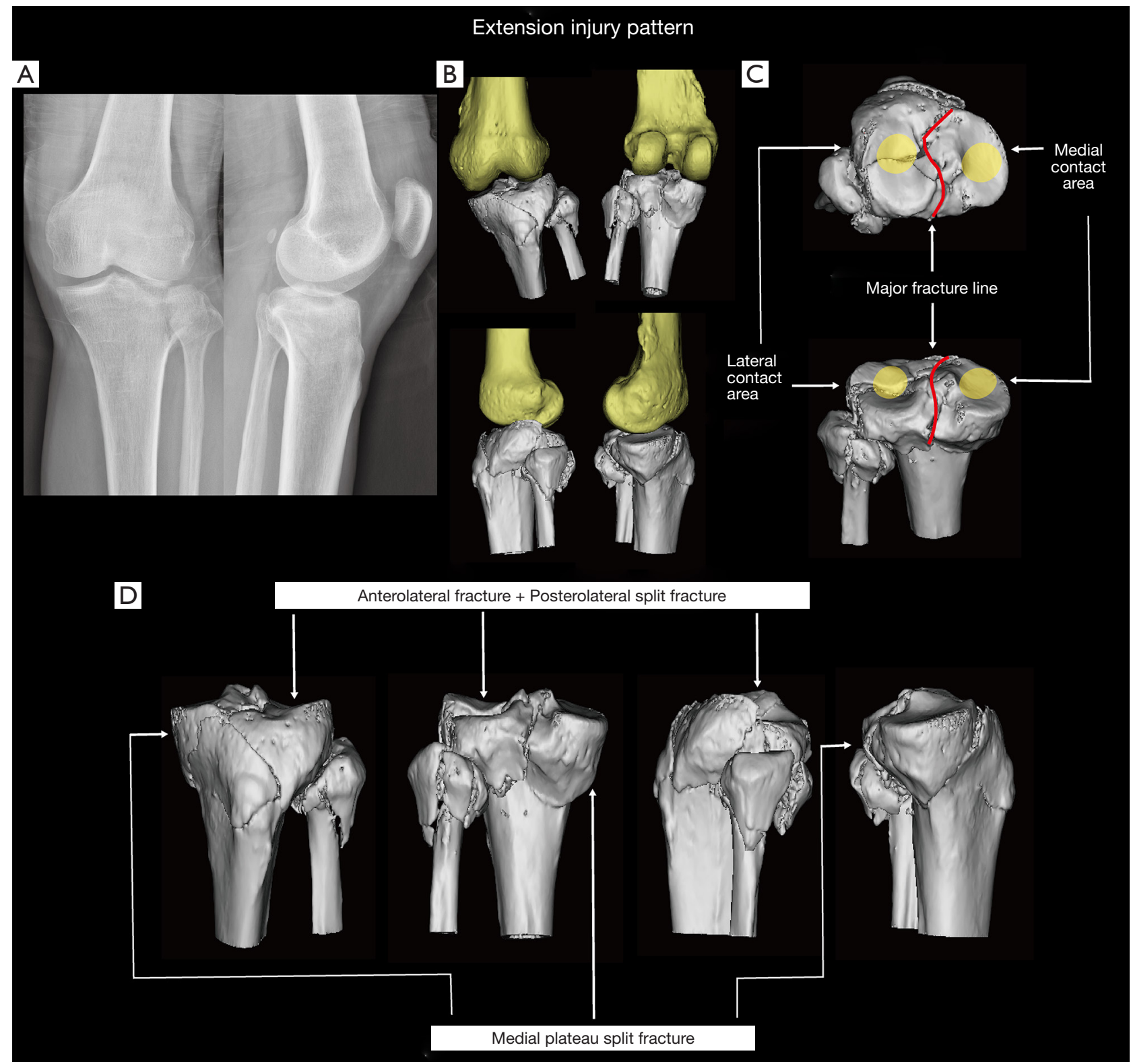

Figure 5 Extension injury pattern. (A) X-ray. (B) Front, back, left and right views of the extension injury pattern. (C) The contact areas and MFL. (D) Fracture characteristics of the extension injury pattern.

$\mathrm{P}=0.004)$. With tibial flexion and external rotation, the lateral contact area was located in the anterolateral quarter plateau, anterior to the fibular head, and the medial contact area was located in the posterior area of the medial plateau, which caused the MFL to be oriented from the anterolateral to the posteromedial plateau (MFLA, $148.26^{\circ}$ ).

A lateral fracture was mainly compressed in the anterolateral part, and the morphology of a posterolateral plateau fracture was mainly split but not comminuted. In the medial plateau, a fracture line located between the attachment of the posterior cruciate ligament and the collateral medial ligament and a posterior medial plateau fracture was always observable (Figure 7).

\section{Flexion-neutral injury pattern}

In total, $17 \mathrm{TPF}$ were in the flexion position $\left(-39.85^{\circ}\right.$ in the sagittal plane) with no obvious rotation $\left(1.74^{\circ}\right.$ in 


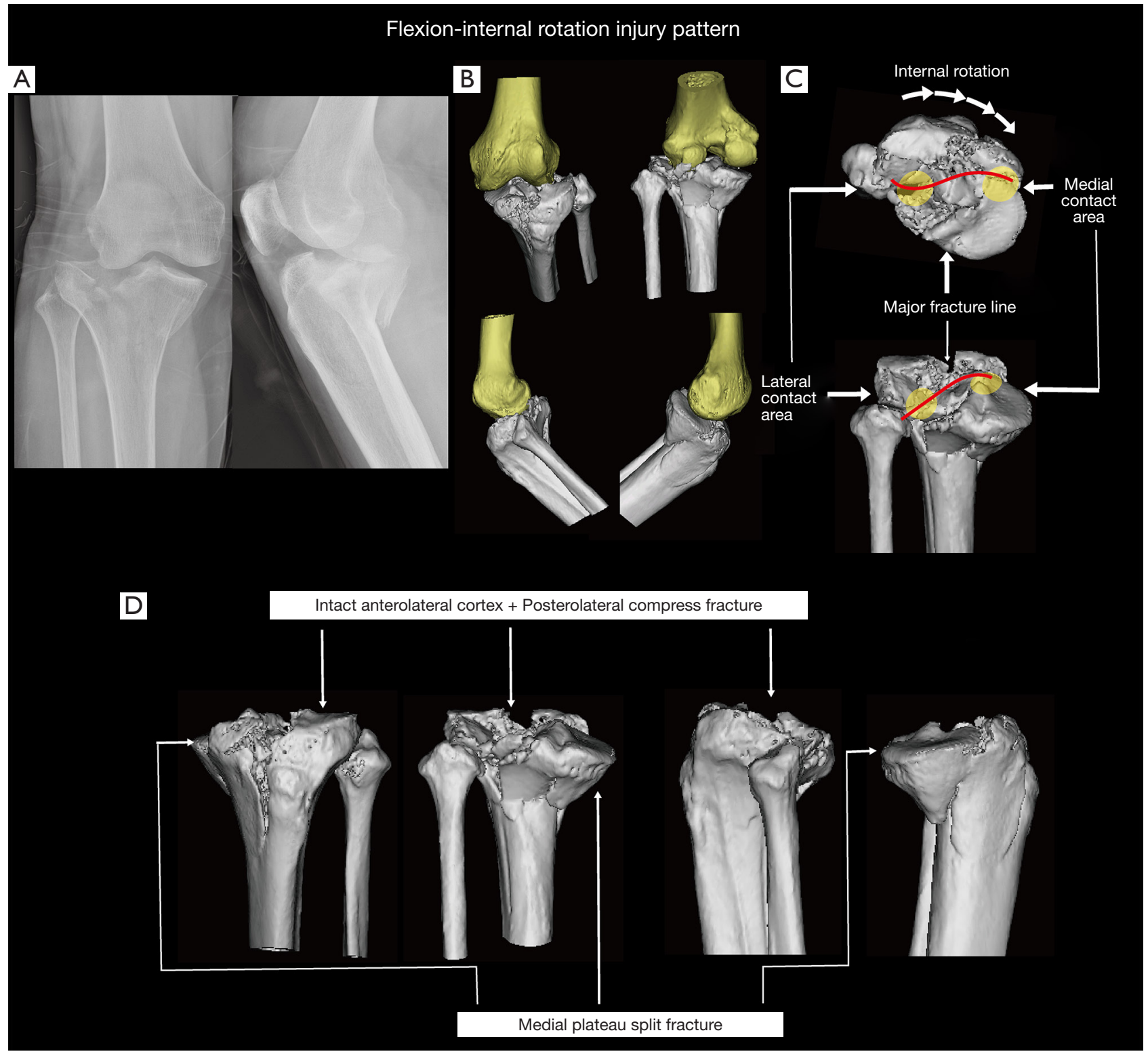

Figure 6 Flexion-internal rotation injury pattern. (A) X-ray. (B) Front, back, left and right views of the flexion-internal rotation injury pattern. (C) The contact areas and MFL. (D) Fracture characteristics of the flexion-internal rotation injury pattern.

the axial plane) regardless of whether the varus or valgus subpattern was observed $\left(-0.41^{\circ}\right.$ in the coronal plane). The lateral and medial contact areas were both located in the posterior quarters of the plateau, and intercondylar eminence avulsion fracture and anterior tibial dislocation were common in this pattern. The MFLs were classified as minor oblique (MFLA, $16.21^{\circ}$ ) from the posterolateral (near the fibular head) to the posteromedial (posterior to the medial collateral ligament attachment) plateau.

Lateral fractures were mainly compressed in the posterior part of the lateral tibial plateau with the intact cortex of the anterior plateau, and the posterolateral fracture morphology was comminuted. The fracture line and fragment in the medial plateau were both located posterior to the medial 


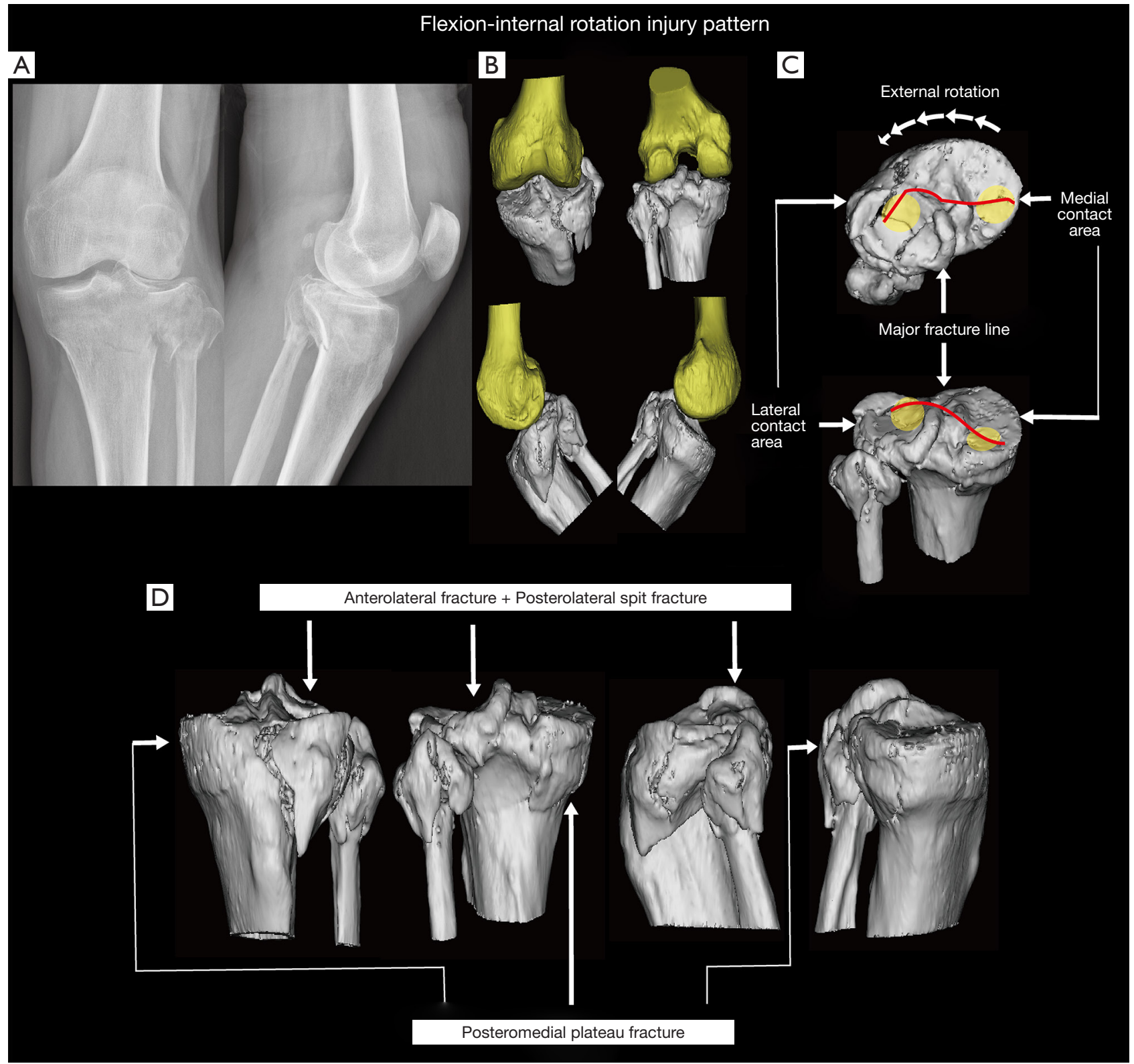

Figure 7 Flexion-external rotation injury pattern. (A) X-ray. (B) Front, back, left and right views of flexion-external rotation injury pattern. (C) The contact areas and MFL. (D) Fracture characteristics of the flexion-external rotation injury pattern.

collateral ligament (Figure 8).

\section{Discussion}

In this study, computer-assisted 3D CT technology was developed to simulate the injury patterns of 171 complex TPFIPs, and a modified fracture mapping method was applied to depict the fracture lines to improve our understanding of this intra-articular injury. After quantitative and qualitative analyses, four main recurrent injury patterns were observed: extension, flexion-internal rotation, flexion-external rotation, and flexion-neutral. The MFL orientations and fracture characteristics significantly differed between the injury patterns according to both statistical and visual comparisons.

The injury patterns of fractures, especially complex 


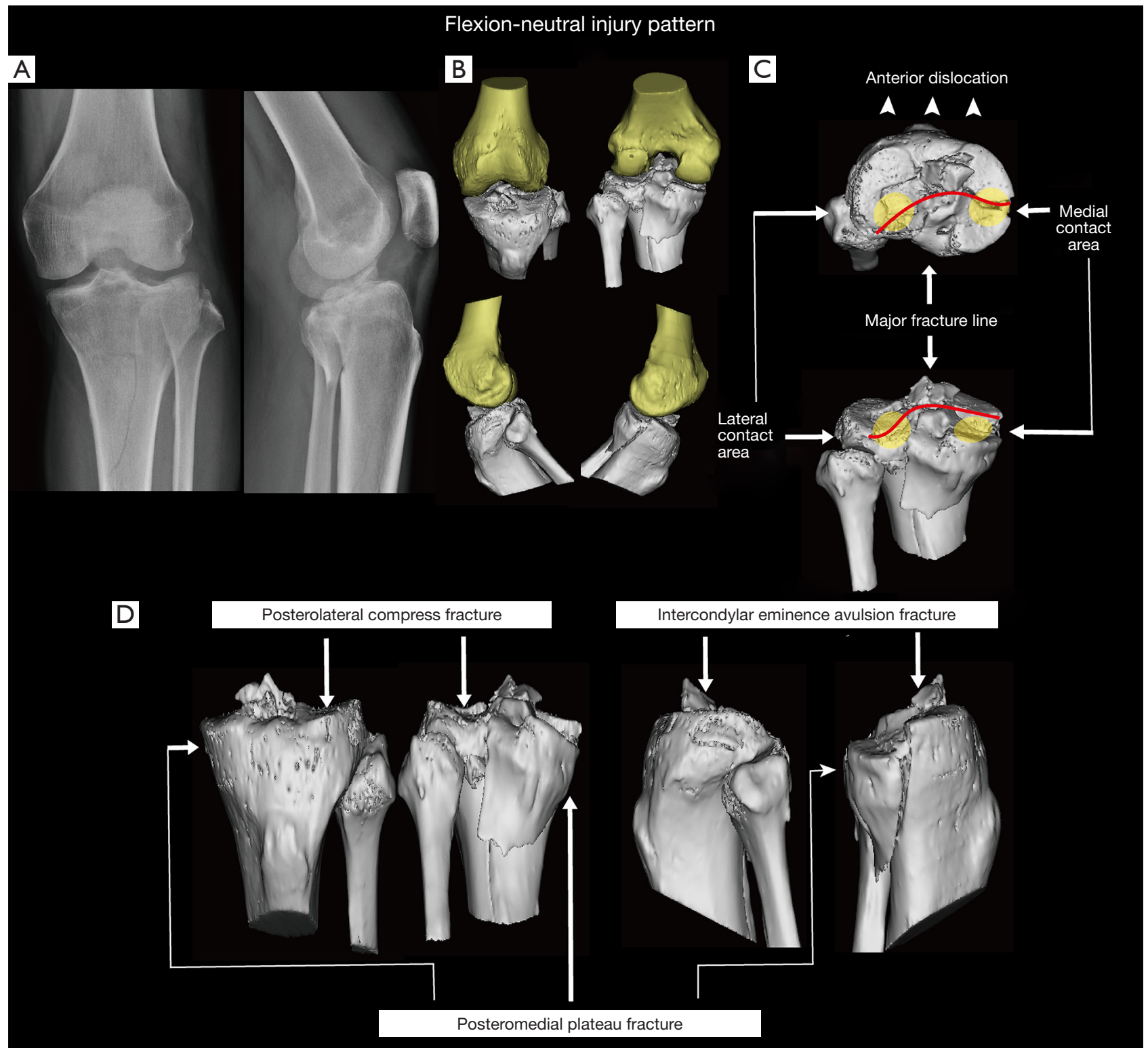

Figure 8 Flexion-neutral rotation injury pattern. (A) X-ray. (B) Front, back, left and right views of the flexion-neutral rotation injury pattern. (C) The contact areas and MFL. (D) Fracture characteristics of the flexion-neutral rotation injury pattern.

TPFs, are difficult to investigate and simulate. The updated Schatzker classification system uses the 3D CT method to demonstrate the TPF morphology and deduce the injury pattern. Wang utilized the 3-column concept to interpret the fracture mechanism and proposed a fixation strategy. These injury patterns are all deduced from the fracture morphology, which requires abundant clinical experience. Recently, Xie et al. (14) demonstrated an injury pattern- related classification system for TPFs with $3 \mathrm{D}$ fracture mapping. In their research, the injury patterns were developed according to 2D CT images of fractures, and the fracture morphology was demonstrated in a $3 \mathrm{D}$ context. They classified the injury patterns into six types based on the tibial articular surface tilt angle in the sagittal plane (hyperextension, extension or flexion) and coronal plane (varus or valgus). However, they ignored the vector in the 
axial plane, which is difficult to assess in 2D CT views; thus, this analysis was not comprehensive. In the current research, we utilized the $3 \mathrm{D}$ simulation method to reconstruct the injury pattern by moving the $3 \mathrm{D}$ tibial model to match the articular surfaces between the tibia and femoral condyle. Next, the injury pattern was demonstrated quantitatively with the vector in the sagittal, coronal, and axial planes. Compared with those of previous studies, the injury pattern identified in the $3 \mathrm{D}$ view was intuitive and visible, and less experience was needed to determine the pattern. A quantitative analysis and comparison of injury patterns was first proposed in the current study. Articular surface matching was performed manually, and the determination of the tibial position seemed to be subjective. However, the optimal injury pattern for every fracture is subject to certain rules, and orthopedic surgeons need to adjust the tibial position to achieve the optimal match in not only the $3 \mathrm{D}$ view but also the sagittal, coronal, and axial views. During matching, the tibial reposition in the three planes was coordinated by linkage, and the final injury pattern was unique and a result of repeat adjustments. We believe that this is a reliable and comprehensive method to interpret the injury patterns of complex articular fractures.

In the current study, 82 TPFIPs (47.95\%) showed a rotation injury pattern, and this number was underestimated in previous studies. The classic injury mechanism of TPFs is a valgus or varus force that causes a fracture, but this mechanism cannot explain the fracture of the posterior part. Luo (5) proposed the inclusion of the posterior column of the tibial plateau and hypothesized that fractures in this area are caused by the loading of a force onto the knee while in flexion; this injury pattern has been confirmed by biomechanical research (15). However, the kinematics of the knee exist in not only the coronal and sagittal planes but also the axial plane (16). Suganuma observed a maximum $35^{\circ}$ external rotation and $25^{\circ}$ internal rotation in $90^{\circ}$ of knee flexion (17). The pivot shift phenomenon $(18,19)$, which is the mechanism of noncontact anterior crucial ligament injury, shows a tibial internal rotation pattern, as demonstrated by the bone bruising located on both the lateral femoral condyle and the posterolateral tibial plateau on MRI (20-22). To the best of our knowledge, no studies have reported the rotation injury pattern for TPFs to date, which may be due in part to the difficulties associated with inferring the position and orientation of the knee at the time of injury with routine imaging examinations.

Fracture characteristics can be illustrated by fracture mapping techniques $(12,23,24)$, which are widely used to depict complex fracture morphologies in a simpler form for better comprehension. Molenaars (7) used this method to demonstrate TPFs, but the results were qualitative and subjective. Similarly, we found that the fracture characteristics in the extension-valgus pattern were consistent with the "lateral split fragment" feature, and the fracture characteristics in the flexion-internal rotation pattern were consistent with the "posteromedial fragment" feature. In contrast to the method used by Molenaars, we herein quantitatively analyzed the MFLAs of four injury patterns, which were found to be significantly different but not indistinguishable. Hence, the injury pattern can be easily diagnosed according to the MFL orientation, and vice versa.

The posterolateral fragment found in this study exhibited two major morphology types, split and depression, which is consistent with the findings of previous studies $(15,25)$. The differences are created by the impact locations of the different injury patterns. In the extension injury pattern, the force load on the anterolateral area creates an anterior compression fracture and then extends to the posterior cortex; thus the posterolateral split type of fragment is more common; this pattern is always accompanied by an anterolateral fracture and an anteroposterior MFL orientation. In contrast, in the flexion-internal rotation and flexion-neutral patterns, the lateral impact is directly located on the posterolateral plateau, which mainly creates a posterolateral depression type of fragment, and the anterolateral plateau remains intact. Consistent with the findings of the present study, a biomechanical experiment conducted by Zhu (15) proposed a depression fragment type with a posterior impact location and a split fragment type with an anterior impact location. Similarly, Chen (26) also demonstrated these two posterolateral fracture patterns in the clinic. According to the present study, an isolated posterolateral fracture exists in only the flexioninternal rotation injury pattern, with a direct impact on the posterolateral plateau, and a posterolateral fracture may accompany an anterolateral fracture (extension injury pattern), medial fracture (flexion-internal rotation injury pattern), or posteromedial and intercondylar eminence avulsion fracture (flexion-neutral injury pattern).

Approximately one-third of bicondylar TPFs and onehalf of medial TPFs have an identifiable posteromedial fragment $(9,27,28)$. Similar to the findings of Barei (27) and Yang (9), three types of posteromedial TPF lines and fracture morphologies were observed in this study. In the extension pattern, the femoral condyles impact the center 
of the medial plateau, which causes the anteroposterior MFL, and a subfracture line with a parallel orientation and a posteromedial fragment are created with continuing force. In the flexion-neutral injury pattern, an anterior dislocation force on the flexed knee without rotation causes an eminent avulsion fracture and posterior plateau impingement; thus, the oblique fracture line from the medial to the posterolateral plateau always accompanies a posterolateral compression fracture and posteromedial split fragment. This unique fracture-dislocation pattern is uncommon, as this pattern was observed in 17 of 171 fractures (9.94\%) in the current study and in 6 of 57 (10.52\%) fractures in the report by Barei (27). Consistent with the assumption of Connolly (29), a posteromedial fragment was also found in the flexion-external rotation injury pattern with the third type of fracture line orientation, i.e., oblique from the anterolateral to the posteromedial plateau, and with anterolateral plateau fractures.

We believe that these findings are important for not only assessing the injury pattern but also planning the surgical strategy. Different injury patterns and fracture characteristics require different surgical strategies. Reversing and neutralizing the injury pattern while reducing the fracture is helpful for fracture fixation. For example, surgeons can reduce a fracture with a flexionexternal rotation valgus injury pattern under extension, internal rotation, and varus conditions. In our preliminary research, fracture reduction was achieved easily with traction in the reverse injury pattern position. Different surgical approaches and fixations should be chosen for varying patterns of injury. The posterolateral fragment in the flexion-external rotation injury pattern is depressed, and a bone graft is needed to support the articular surface with a posterior surgical approach. By contrast, the split posterolateral fragment in the extension injury pattern can be fixed and combined with the anterolateral fragment through an extended lateral approach, and a bone graft may not be necessary.

According to the $3 \mathrm{D}$ view and degree of rotation in the coronal plane observed in this study, Schatzker type IV fractures were found in not only the varus injury pattern but also the valgus injury pattern (Figure 9). The Schatzker type $\mathrm{IV}$ fracture is characterized as a fracture line along the anteroposterior axis, and the injury mechanism is assumed to be a varus force load on the knee (30-32). In the current study, six cases of this classic type IV fracture were found to have the extension-varus injury pattern. However, 21 type IV fractures presented a different injury pattern and a fracture line that could not be explained by this injury mechanism. An oblique fracture line oriented from the posterolateral to the anteromedial plateau was observed in the 21 fractures with a flexion-internal rotation pattern, and based on the tibial position on the coronal plane, 3 fractures were classified as the varus subpattern, and 18 fractures were classified as the valgus subpattern. Under a valgus force with tibial flexion and internal rotation, the lateral femoral condyle impacts the posterolateral plateau to form the compression fracture and the fracture line extending to the anteromedial plateau and distally; thus, on anteroposterior $\mathrm{X}$-rays, this type of medial plateau fracture line is lateral to the intercondylar eminence [type $\mathrm{C}$ in Wahlquist's classification system (33)] and accompanies a localized compression fracture of the posterolateral plateau. The morphology of this medial plateau fracture exhibits an inverted pyramid shape with anteromedial and distal tips. The same finding was demonstrated by Molenaars (7), who also observed a posterior cortex fracture on the lateral plateau on the medial fracture map and suggested that Schatzker type IV fractures may not be unicondylar.

Some limitations of this study must be considered. First, this study included only complex TPFs that involved the posterior plateau and did not include all injury patterns and fracture morphologies, such as avulsion fractures and fractures caused by direct injury, or fractures that did not require surgery because they presented a clear injury mechanism that easily enabled a treatment decision. Second, the findings of our research were based on $3 \mathrm{D}$ simulations, and one may argue that the interpretation of injury patterns and fracture maps is subjective. For the complex construction and kinematics of the knee, the injury pattern is difficult to reproduce precisely. The fracture characteristics simulated in cadaveric experiments are somewhat different from actual fractures, and patients have difficulty recalling and demonstrating the injury pattern or the knee position in the three planes. The injury pattern hypothesis from the fracture characteristics is more subjective and requires extensive experience. The $3 \mathrm{D}$ method proposed herein for simulating injury patterns is feasible and subject to specific rules. In this study, experienced orthopedic surgeons perform the matching, and the match is achieved via both the $3 \mathrm{D}$ and $2 \mathrm{D}$ views (axial, sagittal, and coronal planes). The final tibial position and injury pattern are less subjective and observer-dependent. We believe that further biomechanical investigations will strengthen the evidence presented herein. Third, our conclusion that injury patterns of anterior dislocation are associated with TPFs is preliminary due to 


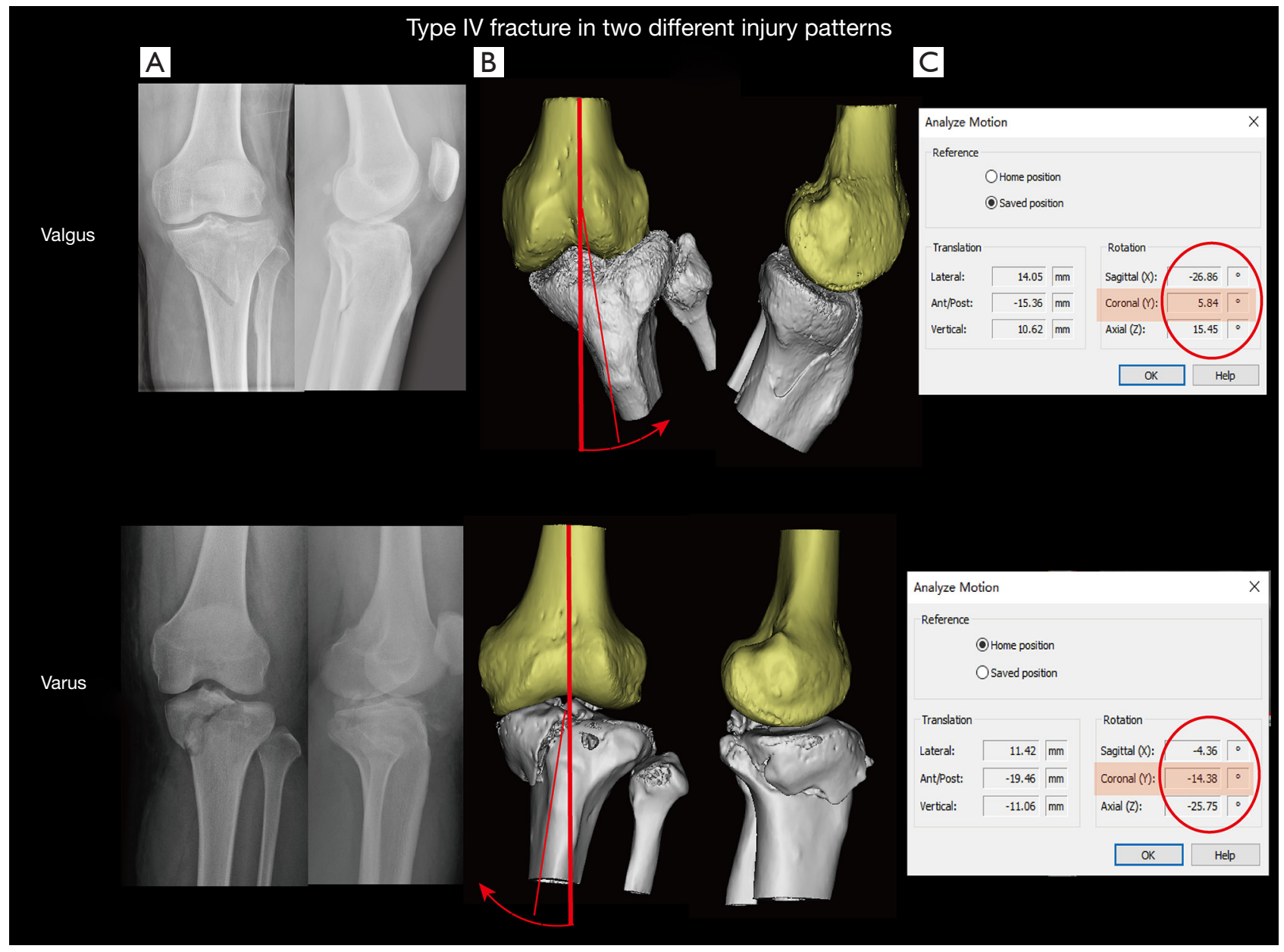

Figure 9 Type IV fractures with two different injury patterns. One type IV tibial plateau fracture exhibited a flexion-internal rotation injury pattern and valgus injury subpattern (9-valgus), and another type IV tibial plateau fracture exhibited an extension injury pattern and varus injury subpattern (9-varus). (A) X-rays of the two TPFs. (B,C) 3D CT injury pattern simulation and analysis of the two TPFs. In the coronal plane, the degree of knee motion differed $\left(5.84^{\circ}\right.$, valgus; $-14.38^{\circ}$, varus).

the complexity of the kinematics and biomechanics of the knee, and the fracture dislocation injury mechanisms are still unclear. Hence, we may further investigate these mechanisms in the future.

In conclusion, we elucidated the injury patterns and fracture characteristics of complex TPFIPs by a $3 \mathrm{D}$ simulation method and fracture mapping in combination with statistical measurements for 171 TPFs. Four main injury patterns were found in this study and can be identified simply by the major fracture line, fracture location and characteristics. The fracture characteristics, categorized by injury patterns, may help to improve observer agreement in clinical studies and may be useful in daily practice as an augmentation to classification systems.

\section{Acknowledgments}

We thank American Journal Experts (AJE) for editing the English language in this manuscript.

Funding: None.

\section{Footnote}

Reporting Checklist: The authors have completed the STROBE reporting checklist. Available at http://dx.doi. org/10.21037/atm-20-5043

Data Sharing Statement: Available at http://dx.doi. org/10.21037/atm-20-5043 


\section{Page 16 of 17}

Peer Review File: Available at http://dx.doi.org/10.21037/ atm-20-5043

Conflicts of Interest: All authors have completed the ICMJE uniform disclosure form (available at http://dx.doi. org/10.21037/atm-20-5043). The authors have no conflicts of interest to declare.

Ethical Statement: The authors are accountable for all aspects of the work in ensuring that questions related to the accuracy or integrity of any part of the work are appropriately investigated and resolved. The study was conducted in accordance with the Declaration of Helsinki (as revised in 2013) and was approved by the Ethics Committee of the Third Hospital of Hebei Medical University (IRB protocol \#2019-036-1). Informed consent was waived because of the retrospective nature of the study.

Open Access Statement: This is an Open Access article distributed in accordance with the Creative Commons Attribution-NonCommercial-NoDerivs 4.0 International License (CC BY-NC-ND 4.0), which permits the noncommercial replication and distribution of the article with the strict proviso that no changes or edits are made and the original work is properly cited (including links to both the formal publication through the relevant DOI and the license). See: https://creativecommons.org/licenses/by-nc-nd/4.0/.

\section{References}

1. Bhattacharyya T, McCarty LP 3rd, Harris MB, et al. The posterior shearing tibial plateau fracture: treatment and results via a posterior approach. J Orthop Trauma 2005;19:305-10.

2. Fakler JK, Ryzewicz M, Hartshorn C, et al. Optimizing the management of Moore type I postero-medial split fracture dislocations of the tibial head: description of the Lobenhoffer approach. J Orthop Trauma 2007;21:330-6.

3. Lobenhoffer P, Gerich T, Bertram T, et al. Particular posteromedial and posterolateral approaches for the treatment of tibial head fractures. Der Unfallchirurg. 1997;100:957-67.

4. Chang SM, Zheng HP, Li HF, et al. Treatment of isolated posterior coronal fracture of the lateral tibial plateau through posterolateral approach for direct exposure and buttress plate fixation. Arch Orthop Trauma Surg 2009;129:955-62.

5. Luo CF, Sun H, Zhang B, Zeng BF. Three-column fixation

\section{Pan et al. Injury pattern and mapping of tibial plateau fractures}

for complex tibial plateau fractures. J Orthop Trauma 2010;24:683-92.

6. Chang SM, Hu SJ, Zhang YQ, et al. A surgical protocol for bicondylar four-quadrant tibial plateau fractures. Int Orthop 2014;38:2559-64.

7. Molenaars RJ, Mellema JJ, Doornberg JN, et al. Tibial Plateau Fracture Characteristics: Computed Tomography Mapping of Lateral, Medial, and Bicondylar Fractures. J Bone Joint Surg Am 2015;97:1512-20.

8. Kfuri M, Schatzker J. Revisiting the Schatzker classification of tibial plateau fractures. Injury 2018;49:2252-63.

9. Yang G, Zhu Y, Luo C, et al. Morphological characteristics of Schatzker type IV tibial plateau fractures: a computer tomography based study. Int Orthop 2012;36:2355-60.

10. Zeng ZM, Luo CF, Putnis S, et al. Biomechanical analysis of posteromedial tibial plateau split fracture fixation. Knee 2011;18:51-4.

11. Doornberg JN, Rademakers MV, van den Bekerom MP, et al. Two-dimensional and three-dimensional computed tomography for the classification and characterisation of tibial plateau fractures. Injury 2011;42:1416-25.

12. Armitage BM, Wijdicks CA, Tarkin IS, et al. Mapping of scapular fractures with three-dimensional computed tomography. J Bone Joint Surg Am 2009;91:2222-8.

13. Kim HY, Kim KJ, Yang DS, et al. Screw-Home Movement of the Tibiofemoral Joint during Normal Gait: ThreeDimensional Analysis. Clin Orthop Surg 2015;7:303-9.

14. Xie X, Zhan Y, Wang Y, et al. Comparative Analysis of Mechanism-Associated 3-Dimensional Tibial Plateau Fracture Patterns. J Bone Joint Surg Am 2020;102:410-8.

15. Zhu Y, Meili S, Dong MJ, et al. Pathoanatomy and incidence of the posterolateral fractures in bicondylar tibial plateau fractures: a clinical computed tomography-based measurement and the associated biomechanical model simulation. Arch Orthop Trauma Surg 2014;134:1369-80.

16. Robertson DD, Debski RE, Almusa E, et al. Knee joint biomechanics: relevance to imaging. Semin Musculoskelet Radiol 2003;7:43-58.

17. Suganuma J, Ohkoshi T. Association of internal rotation of the knee joint with recurrent subluxation of the lateral meniscus. Arthroscopy 2011;27:1071-8.

18. Fetto JF, Marshall JL. Injury to the anterior cruciate ligament producing the pivot-shift sign. J Bone Joint Surg Am 1979;61:710-4.

19. Neumann RD. Traumatic knee injuries. Prim Care 1992;19:351-76.

20. Hayes CW, Brigido MK, Jamadar DA, et al. Mechanismbased pattern approach to classification of complex injuries 
of the knee depicted at MR imaging. Radiographics 2000;20:S121-34.

21. Sanders TG, Medynski MA, Feller JF, et al. Bone contusion patterns of the knee at MR imaging: footprint of the mechanism of injury. Radiographics 2000;20:S135-51.

22. Costa-Paz M, Muscolo DL, Ayerza M, et al. Magnetic resonance imaging follow-up study of bone bruises associated with anterior cruciate ligament ruptures. Arthroscopy 2001;17:445-9.

23. Mellema JJ, Doornberg JN, Dyer GS, et al. Distribution of coronoid fracture lines by specific patterns of traumatic elbow instability. J Hand Surg Am 2014;39:2041-6.

24. Zhang X, Zhang Y, Fan J, et al. Analyses of fracture line distribution in intra-articular distal radius fractures. Radiol Med 2019;124:613-9.

25. Zhai Q, Luo C, Zhu Y, et al. Morphological characteristics of split-depression fractures of the lateral tibial plateau (Schatzker type II): a computer-tomography-based study. Int Orthop 2013;37:911-7.

26. Chen HW, Chen CQ, Yi XH. Posterior tibial plateau fracture: a new treatment-oriented classification and surgical management. Int J Clin Exp Med 2015;8:472-9.

27. Barei DP, O'Mara TJ, Taitsman LA, et al. Frequency and fracture morphology of the posteromedial fragment

Cite this article as: Pan S, Peng AQ, Hu YN, Wang S, Zhang YL, Wang Y. Injury pattern simulation and mapping of complex tibial plateau fractures that involve the posterior plateau with three-dimensional computed tomography. Ann Transl Med 2021;9(4):302. doi: 10.21037/atm-20-5043 in bicondylar tibial plateau fracture patterns. J Orthop Trauma 2008;22:176-82.

28. Yang G, Zhai Q, Zhu Y, et al. The incidence of posterior tibial plateau fracture: an investigation of 525 fractures by using a CT-based classification system. Arch Orthop Trauma Surg 2013;133:929-34.

29. Connolly JF. The posterior shearing tibial plateau fracture: treatment and results via a posterior approach. J Orthop Trauma 2005;19:508; author reply 508 .

30. Kennedy JC, Bailey WH. Experimental tibial-plateau fractures. Studies of the mechanism and a classification. J Bone Joint Surg Am 1968;50:1522-34.

31. Schatzker J, McBroom R, Bruce D. The tibial plateau fracture. The Toronto experience 1968--1975. Clin Orthop Relat Res 1979;(138):94-104.

32. Wang Y, Luo C, Zhu Y, et al. Updated Three-Column Concept in surgical treatment for tibial plateau fractures - A prospective cohort study of 287 patients. Injury 2016;47:1488-96.

33. Wahlquist M, Iaguilli N, Ebraheim N, et al. Medial Tibial Plateau Fractures: a New Classification System. J Trauma 2007;63:1418-21. 